REGARDS

SUR L'ECONOMIE ALLEMAND

BULLETIN ECONOMIQUE DU CIRAC
Regards sur l'économie allemande

Bulletin économique du CIRAC

$102 \mid 2011$

Varia

\title{
Un ralentissement prévisible
}

Isabelle Bourgeois

\section{OpenEdition}

Journals

Édition électronique

URL : http://journals.openedition.org/rea/4321

DOI : 10.4000/rea.4321

ISBN : 978-2-8218-1134-8

ISSN : 1965-0787

Éditeur

CIRAC

Édition imprimée

Date de publication : 31 octobre 2011

Pagination : 3-4

ISSN : 1156-8992

Référence électronique

Isabelle Bourgeois, «Un ralentissement prévisible», Regards sur l'économie allemande [En ligne], 102 | octobre 2011, mis en ligne le 04 novembre 2011, consulté le 15 septembre 2020. URL : http:// journals.openedition.org/rea/4321 


\section{Un ralentissement prévisible}

«La crise européenne de la dette pèse sur la conjoncture allemande ». Tel est le titre du rapport d'automne présenté par les Instituts de conjoncture le 13 octobre. Les perspectives de l'économie mondiale se sont assombries, et l'Europe est affectée par la crise de la dette souveraine. L'économie allemande s'en ressent nécessairement, la faiblesse de la demande mondiale et européenne bridant le moteur des exportations. S'ajoutent à l'évolution du contexte l'expiration des programmes conjoncturels allemands, ainsi que diverses hausses dont celles des matières premières, des produits pétroliers ou des cotisations. Après une forte croissance depuis le début de l'année, les activités devraient donc ralentir à l'approche de l'hiver. Au total, les Instituts prévoient une croissance de 2,9\% en 2011, et de 0,8\% seulement en 2012. Mais l'exercice de la prévision conjoncturelle est particulièrement difficile en ce moment, tant les incertitudes sont grandes, et les Instituts soulignent que d'importantes variations (récession ou à l'inverse forte reprise) ne sont pas à exclure. Le sort de l'économie allemande dépendra de la progression du dossier européen et de la capacité des responsables politiques de l'UE à restituer, par les décisions qu'ils prendront, le climat de confiance des acteurs économiques.

\begin{tabular}{|c|c|c|c|c|}
\hline \multicolumn{5}{|c|}{ Prévisions du rapport d'automne 2011 du Groupe de travail des Instituts économiques } \\
\hline & 2009 & 2010 & 2011 & 2012 \\
\hline \multicolumn{5}{|c|}{ variation en \% par rapport à l'année précédente (données CVS) } \\
\hline$P M: P I B$ de la zone $€$ & $-4,2$ & 1,8 & 1,5 & 0,4 \\
\hline Consommation privée & $-0,1$ & 0,6 & 1,2 & 0,9 \\
\hline Consommation publique & 3,3 & 1,7 & 1,1 & 1,1 \\
\hline construction & $-3,0$ & 2,2 & 5,8 & 1,3 \\
\hline autres & 0,6 & 4,7 & 4,3 & 4,9 \\
\hline Demande intérieure & $-2,6$ & 2,4 & 2,6 & 1,2 \\
\hline Exportations & $-13,6$ & 13,7 & 7,8 & 2,9 \\
\hline Importations & $-9,2$ & 11,7 & 7,7 & 4,1 \\
\hline Prix à la consommation (2005 = indice 100) & 0,4 & 1,1 & 2,3 & 1,8 \\
\hline Déficit public (en milliards $€$ ) & $-76,1$ & $-106,0$ & & \\
\hline Actifs (en millions) & 40,362 & 40,553 & 40,082 & 41,274 \\
\hline Taux de chômage en \% (Agence de Nuremberg) & 8,1 & 7,7 & 7,0 & 6,7 \\
\hline Chômeurs indemnisés (en millions) & 3,415 & 3,238 & 2,968 & 2,815 \\
\hline
\end{tabular}

Source des données: Gemeinschaftsdiagnose Herbst 2011 (13-10-2011). Le groupe de travail comprend 4 binômes d'instituts durant la période automne 2010/printemps 2013 : ifo (Munich) + KOF (Zurich), IfW (Kiel) + ZEW (Mannheim), IWH (Halle) + Kiel Economics (Kiel) et RWI (Essen) + IHS (Vienne). Les prévisions prennent pour hypothèse notamment un prix moyen du baril de Brent de $113 \$$ en 2011 et 2012 , une parité $1,38 \$=1 €$, et une hausse du commerce mo sont que partiellement comparables a celles des rapports précédents, les instituts

Malgré ces risques, les entreprises se montrent foncièrement confiantes. L'indice ifo du climat des affaires (le dernier remonte à la fin septembre) est légèrement orienté à la baisse depuis l'été, mais cela concerne surtout, et en toute logique, les prévisions pour les six mois à venir ; la situation présente est jugée favorablement. II n'y a pas signe de restriction du crédit bancaire. Les entreprises industrielles comptent toujours embaucher bien que leurs carnets de commande se remplissent un peu moins vite. Mais elles sont plus prudentes et tendent à ajourner notamment l'extension de leurs capacités, ce dont se ressent le climat des affaires dans le BTP, du moins en ce qui concerne ses prévisions. Quant au commerce, il est résolument optimiste - surtout le commerce de détail qui avait dernièrement enregistré une hausse de ses activités. Cela concorde avec les calculs de Destatis qui, au vu de l'évolution depuis la fin 2009, constatait que l'économie allemande "poursuit sur la voie de la reprise au-delà du $2^{\text {ème }}$ trimestre " (communiqué du 01-09-2011). Pour sa part, la Fédération de l'industrie BDI estime " qu'il n'existe aucune raison fondée pour que l'économie réelle entre en récession " (communiqué du 13-10-2011).

II est vrai que la perspective d'un ralentissement conjoncturel n'est pas une surprise : celui-ci était prévu depuis longtemps, la date d'expiration des programmes conjoncturels étant connue, et les entreprises allemandes se tenant régulièrement
Les entreprises sont confiantes

Elles ont anticipé le ralentissement à venir 
Confiance des consommateurs

Une seule angoisse : la stabilité monétaire informées de l'évolution du contexte européen et mondial. Elles s'y préparent donc depuis des mois, comme l'explique par exemple le quotidien Handelsblatt dans un long dossier paru sous le titre évocateur: "Sans peur vers la récession 》 (06-102011). En réalité, la récession passée a aiguisé leur sens de la prévention du risque, les incitant à reconstituer ou augmenter leurs capitaux propres afin de n'avoir à recourir que le moins possible au crédit bancaire, à optimiser la gestion des flux et à affiner encore leur vaste éventail d'instruments de flexibilité interne (voir REA 101/2011), voire à miser massivement sur cette variable d'ajustement qu'est outre-Rhin le travail intérimaire. «Les indicateurs hard'... confirment les indicateurs 'soft' » que sont les indices de confiance, estimait de son côté la Bundesbank dans son rapport de septembre sur l'état de la conjoncture allemande.

Car les consommateurs aussi ont le moral. La récession n'avait que peu affecté l'emploi, et les embauches ont repris. Le nombre de chômeurs est tombé en septembre à son plus bas niveau depuis... 1991 ; l'Agence de Nuremberg en dénombrait alors moins de 2,8 millions. De janvier à juillet, le nombre d'emplois soumis à cotisations sociales a augmenté de 447000 , dont la moitié à temps plein. Selon les Instituts, le marché de l'emploi devrait rester robuste malgré l'arrivée massive de jeunes sur le marché du travail du fait du raccourcissement d'un an de l'enseignement secondaire et de la suppression du service militaire. Quant aux salaires conventionnels, ils avaient été en hausse de 1,5\% en moyenne au premier semestre 2011 , les salaires effectifs même de $2,9 \%$ du fait des primes versées par nombre d'entreprises ou de l'accroissement du volume des heures supplémentaires. Les accords de branche, dont la plupart couvrent 2011 et 2012, prévoient une hausse des salaires de 1,8\% en moyenne cette année et de 2,5 \% l'an prochain.

Si la consommation des ménages est restée à un niveau somme toute modeste dans ce contexte porteur, c'est que les revenus du patrimoine et ceux issus de l'activité entrepreneuriale ont, eux, nettement baissé. Les patrons du Mittelstand ont apporté leur contribution à la sortie de crise. Par ailleurs, le pouvoir d'achat des ménages est grevé par la flambée du fuel domestique, des produits alimentaires, d'une série de prix administrés, de la hausse arithmétique du revenu imposable du fait de l'inflation, et par les mesures de politique fiscale adoptées dans le contexte de la réduction du déficit (voir la rubrique «Actualité »). Mais ce qui incite le plus les Allemands à se montrer prudents dans leurs achats ou leurs projets d'acquisition de biens autres que durables, c'est l'incertitude qui règne actuellement quant à la stabilité de la monnaie.

CONFIANCE : C'EST LÀ LE MOT CLÉ DE L'ÉVOLUTION DE LA CONJONCTURE. Les fondamentaux de l'économie allemande sont robustes : l'industrie est toujours aussi dynamique, innovante, réactive à la demande, bien armée pour investir le cas échéant et préserver l'équilibre de la balance commerciale ; la consommation tient bon ; et, pour couronner le tout, les responsables politiques allemands font preuve de détermination dans la poursuite de la consolidation des finances publiques - même si cet effort va tasser le PIB de 0,8 \% en 2012 (Instituts).

Dès lors, les préconisations des Instituts portent surtout sur la gestion de la crise de la dette souveraine par les Etats de la zone Euro. A l'échelon national, ils appellent le gouvernement à respecter strictement la 'règle d'or' constitutionnelle de compression du déficit - dans l'intérêt de l'Allemagne, mais bien plus encore "pour donner le signal dans la crise de la dette souveraine ». Car ce qui importe le plus, ce n'est pas tant la vitesse à laquelle les Etats réduiront leur dette que la "crédibilité de leur engagement sur la voie d'une consolidation durable». Ils appellent donc aussi le gouvernement à " ne pas céder à la pression » de certains Etats ou du FMI, voire du Parlement européen, qui préconisent l'adoption d'un plan de relance de la consommation. Seul le gage de confiance collectif qu'est une gestion saine des finances publiques permettra le retour durable de la croissance au sein de la zone Euro et dans sa principale 'locomotive'. " L'Allemagne doit rester l'ancre de stabilité et de croissance en Europe ", affirmait le ministre fédéral de l'Economie en réaction au rapport des Instituts. Bien que rituelle, cette formule n'en est pas moins un appel pressant aux partenaires de la RFA dans la zone Euro à s'accorder d'urgence sur les réformes institutionnelles. Elles sont la condition sine qua non aussi de la stabilité de sa compétitivité, et partant de celle de ses partenaires.

l. Bourgeois (14-10-2011) 